\title{
A SUFFICIENT CONDITION FOR NON-SOFICNESS OF HIGHER-DIMENSIONAL SUBSHIFTS
}

\author{
STEVE KASS AND KATHLEEN MADDEN
}

(Communicated by Bryna Kra)

\begin{abstract}
A shift space is said to be sofic if it is the factor of a shift of finite type. In one dimension, there are complete characterizations of soficness. There are no characterizations in higher dimensions, and there are few examples of non-sofic $\mathbb{Z}^{d}$ shifts for $d>1$. In this work we give a condition that implies non-soficness in higher-dimensional shift spaces, and we apply it to a variety of examples.
\end{abstract}

\section{BACKGROUND DEFINITIONS}

Let $\mathcal{A}=\{1, \ldots, n\}$ be a finite alphabet, and let $D \subseteq \mathbb{Z}^{d}, d \geq 1$. For $y \in \mathcal{A}^{D}$, $\vec{v} \in D$ and $S \subseteq D$, we will denote the symbol appearing in $y$ at $\vec{v}$ as $y_{\vec{v}}$ and the configuration of symbols appearing in $y$ in the locations in $S$ as $y_{S}$. When $S$ is a one-dimensional subset of $D$ of the form $S=\left\{\vec{a}+k \overrightarrow{e_{i}} \mid 0 \leq k<m\right\}$ for some $1 \leq i \leq d$, we will call configuration $y_{S}$ a word (of length $m$ ).

For $d \geq 1$, the $d$-dimensional, full $n$-shift is the set $\mathcal{A}^{\mathbb{Z}^{d}}$ together with the $\mathbb{Z}^{d}$ action defined by

$$
\sigma_{\vec{v}}(x)_{\vec{w}}=x_{\vec{v}+\vec{w}}
$$

for any $x \in \mathcal{A}^{\mathbb{Z}^{d}}$ and any $\vec{v}, \vec{w} \in \mathbb{Z}^{d}$. A closed shift-invariant subset $X$ of $\mathcal{A}^{\mathbb{Z}^{d}}$ together with the restriction of the $\mathbb{Z}^{d}$-action to $X$ is called a $\mathbb{Z}^{d}$-shift space and is denoted by $\left(X, \mathbb{Z}^{d}\right)$.

Shift spaces can be defined via a set of forbidden configurations. If this set of forbidden configurations can be chosen to be finite, $\left(X, \mathbb{Z}^{d}\right)$ is called a shift of finite type. A shift space $\left(Y, \mathbb{Z}^{d}\right)$ is sofic if and only if it is the image of a continuous, shift commuting map $\phi: X \rightarrow Y$ for some shift of finite type $\left(X, \mathbb{Z}^{d}\right)$.

A matrix shift is defined by zero-one $n \times n$ transition matrices $H_{1}, H_{2}, \ldots, H_{d}$; symbols $j, k \in \mathcal{A}$ are forbidden from occurring adjacent to each other in the $\vec{e}_{i}$ direction if and only if $H_{i}(j, k)=0$.

Up to conjugacy, every shift of finite type is equivalent to a matrix shift and every continuous, shift commuting map $\phi: X \rightarrow Y$ can be taken to be a map on symbols. (See $[\mathrm{LM}]$ for additional background, particularly in the one-dimensional case.) So we may assume that a shift space $\left(Y, \mathbb{Z}^{d}\right)$ is sofic with alphabet $\mathcal{A}^{\prime}$ if and

Received by the editors June 9, 2011 and in revised form, October 26, 2011 and January 9, 2012 .

2010 Mathematics Subject Classification. Primary 37B50; Secondary 37B10.

(C) 2013 American Mathematical Society 
only if there exists a matrix shift $\left(X, \mathbb{Z}^{d}\right)$ with alphabet $\mathcal{A}$ and a map on symbols

$$
\Phi: \mathcal{A} \rightarrow \mathcal{A}^{\prime}
$$

via which the factor map

$$
\phi: X \rightarrow Y
$$

applies $\Phi$ to the individual symbols of a point in $X$. In all that follows, we will refer to both maps as $\phi$. In addition, and without loss of generality, when we refer to a map $\phi$ between shift spaces, we will mean that it is a 1-block code given by a map $\Phi$ on symbols.

When $\left(X, \mathbb{Z}^{d}\right)$ is a shift space and $D \subseteq \mathbb{Z}^{d}$, we say a configuration $C \in \mathcal{A}^{D}$ occurs in $X$ if there exists $x \in X$ with $x_{D}=C$. We say $C$ is allowed if $C$ does not contain any of the defining forbidden configurations. Note that all occurring configurations are necessarily allowed, but allowed configurations might or might not occur.

Special notation for several particular subsets of $\mathbb{Z}^{d}$ will be helpful. Let $B_{k}^{d}$ be the $k^{d}$-rectangular block with "lower left corner" $(1,1, \ldots, 1) \in \mathbb{Z}^{d}$. That is,

$$
B_{k}^{d}=\left\{\left(a_{1}, a_{2}, \ldots, a_{d}\right) \mid 1 \leq a_{i} \leq k \text { for } 1 \leq i \leq d\right\} .
$$

Denote the complement of $B_{k}^{d}$ by $F_{k}^{d}=\mathbb{Z}^{d} \backslash B_{k}^{d}$. When the context is understood, we will refer to $B_{k}^{d}$ and $F_{k}^{d}$ simply as $B_{k}$ and $F_{k}$.

We note that the number of lattice points in $\partial\left(B_{k}\right)$, the boundary of $B_{k}$, is $k^{d}-(k-2)^{d}$, which is less than $2 d \cdot k^{d-1}$. This observation will be useful in the proof of Theorem 2.3 .

The following ideas will also be needed:

Definition 1.1. Let $\left(Y, \mathbb{Z}^{d}\right)$ be a shift space and let $C$ be a configuration on $B_{k}$. The extender set of $C$, denoted $E_{Y}(C)$, is given as follows:

$$
E_{Y}(C)=\left\{y_{F_{k}} \mid y \in Y \text { and } y_{B_{k}}=C\right\} .
$$

The collection of extender sets for all occurring configurations on $B_{k}$ is denoted $\mathcal{E}_{Y}^{k}$ :

$$
\mathcal{E}_{Y}^{k}=\left\{E_{Y}(C) \mid C \text { is an occurring configuration on } B_{k}\right\} .
$$

Because a matrix shift $\left(X, \mathbb{Z}^{d}\right)$ is given by nearest-neighbor transitions, the extender set $E_{X}(C)$ depends only on the configuration on the boundary of $B_{k}$. If $\left(Y, \mathbb{Z}^{d}\right)$ is sofic with $\phi: X \rightarrow Y$, then Claim 1.2 shows that the extender sets of $\left(Y, \mathbb{Z}^{d}\right)$ can be characterized in terms of the extender sets of $\left(X, \mathbb{Z}^{d}\right)$. (The proof of Claim 1.2 is straightforward and is left to the reader.)

Claim 1.2. Let $\left(X, \mathbb{Z}^{d}\right)$ be a matrix shift and let $\left(Y, \mathbb{Z}^{d}\right)$ be sofic with $\phi: X \rightarrow Y$ a 1-block code. Let $C$ be a configuration on $B_{k}$ occurring in $\left(Y, \mathbb{Z}^{d}\right)$. Let $A_{1}$, $A_{2}, \ldots, A_{j}$ be the pre-images under $\phi$ of $C$ that occur in $\left(X, \mathbb{Z}^{d}\right)$. Then

$$
E_{Y}(C)=\bigcup_{1 \leq i \leq j} \phi\left(E_{X}\left(A_{i}\right)\right)
$$

Claim 1.2 tells us that $E_{Y}(C)$ is determined by the configurations on $\partial\left(B_{k}\right)$ of the pre-images of $C$. We will denote these "boundary pre-images" by $B P(C)$. That is,

$$
B P(C)=\left\{x_{\partial\left(B_{k}\right)} \mid x \in X \text { and } \phi(x)_{B_{k}}=C\right\} .
$$




\section{A SUFFICIENT CONDITION FOR NON-SOFICNESS IN HIGHER DIMENSIONS}

For one-dimensional shift spaces, there is a simple characterization of soficness. Let $(Y, \mathbb{Z})$ be a shift space with alphabet $\mathcal{A}$, and let $w$ be a finite word that occurs in $Y$. Define $R_{Y}(w)$ (the right follower set of $w$ ) to be the set of half-infinite configurations that occur in points of $Y$ immediately to the right of $w$. That is,

$$
R_{Y}(w)=\left\{y_{[0, \infty)} \mid w y_{[0, \infty)} \text { occurs in } Y\right\} .
$$

It is well known that the shift space $(Y, \mathbb{Z})$ is sofic if and only if the set

$$
\mathcal{R}_{Y}=\left\{R_{Y}(w) \mid w \text { is a finite occurring word in } Y\right\}
$$

is finite ( $[\mathrm{LM}]$, Theorem 3.2.10). (This is also true for the collection of "left predecessor" sets.)

Informally, the intuition behind this fact is that in one dimension, $(Y, \mathbb{Z})$ is sofic and $\mathcal{R}_{Y}$ is finite exactly when the way in which a word can be extended depends only on a finite amount of hidden state information on each boundary symbol of the word.

There is no equivalent characterization of soficness in higher dimensions, nor is there a single canonical generalization of the characterizing condition. However, we might hope that a finite amount of hidden state information on the boundary symbols of a higher-dimensional configuration $C$ might also be enough to determine $E_{Y}(C)$. So, because $\left|\partial\left(B_{k}\right)\right|=2 d k^{d-1}$ grows with $k$ for $d>1$, it is natural to ask whether there is some condition on the growth rate of $\left|\mathcal{E}_{Y}^{k}\right|($ as $k \rightarrow \infty)$ that might be useful in characterizing the soficness of $\left(Y, \mathbb{Z}^{d}\right)$. A few examples illustrate the idea.

We first observe that if $\left(X, \mathbb{Z}^{d}\right)$ is a shift of finite type with alphabet $\mathcal{A}$, then

$$
\left|\mathcal{E}_{X}^{k}\right| \leq\left|\mathcal{A}^{\partial\left(B_{k}\right)}\right| \leq|\mathcal{A}|^{2 d k^{d-1}} .
$$

Thus in $\mathbb{Z}^{d}$ shifts of finite type, $\log \left(\left|\mathcal{E}_{X}^{k}\right|\right)$ is in $O\left(k^{d-1}\right)$.

In addition, there are several higher-dimensional subshifts $\left(Y, \mathbb{Z}^{d}\right)$ in the literature that are known to be strictly sofic (i.e. sofic but not a shift of finite type) for which $\log \left(\left|\mathcal{E}_{Y}^{k}\right|\right) \in O\left(k^{d-1}\right)$. These include: the two-dimensional connected components odd shift, $Y_{\text {odd }} \subset\{0,1\}^{\mathbb{Z}^{2}}$, where finite connected components of 1 's must be of odd size $([\vec{H},[\mathrm{C})$; the two-dimensional context-free shift ( $\mathrm{LMN}$, Section $2.5)$; and the higher-dimensional even-run shifts contained in $\{0,1\} \mathbb{Z}^{d}$, where any run of 1's in a coordinate direction has even length. (We omit details of these examples, but the interested reader can easily verify that their extender sets have the aforementioned growth condition.)

However, there are also (unpublished) examples of subshifts ([HQ], [P2]) which are strictly sofic but for which the asymptotic growth rate of $\log \left(\left|\mathcal{E}_{X}^{k}\right|\right)$ is greater than $O\left(k^{d-1}\right)$. So a log growth rate for extender sets greater than $O\left(k^{d-1}\right)$ is not by itself enough to imply non-soficness. Our main result, Theorem 2.3 will show that if we can find extender sets that grow more quickly than this and that also overlap in a particular way, then $\left(Y, \mathbb{Z}^{d}\right)$ must be non-sofic. This overlapping condition is described in the following definition.

Definition 2.1. Let $\left\{S_{i}\right\}_{i=1}^{m}$ be a finite sequence of non-empty sets. The sequence $\left\{S_{i}\right\}$ is called union-increasing if the partial unions of the $\left\{S_{i}\right\}$ strictly increase: if for $1 \leq i \leq m, S_{i} \supset \bigcup_{j<i} S_{j}$. 
Before stating Theorem 2.3, we include a lemma which will be useful in the theorem's proof. Lemma 2.2 will identify a condition in a sofic shift $\left(Y, \mathbb{Z}^{d}\right)$ that provides information about the sets $B P(C)$, which we defined earlier. Roughly, Lemma 2.2 states the following: If $y \in Y$ and $C$ is a configuration on $B_{k}$ that occurs in $Y$ but does not "fit" into the middle of $y$ (in place of $y_{B_{k}}$ ), then $B P\left(y_{B_{k}}\right)$ and $B P(C)$ are disjoint.

Lemma 2.2. Let $\left(Y, \mathbb{Z}^{d}\right)$ be a sofic shift space with $\phi: X \rightarrow Y$ where $\left(X, \mathbb{Z}^{d}\right)$ is a matrix shift and $\phi$ is a 1-block code. Assume that $C$ is a configuration on $B_{k}$ that occurs in $\left(Y, \mathbb{Z}^{d}\right)$ and that $y \in Y$ is a point for which $y_{F_{k}} \notin E_{Y}(C)$. Let $x$ be any pre-image of $y$, and write $P=x_{\partial\left(B_{k}\right)}$. Then $P \notin B P(C)$.

Proof. Let $C, y, x$, and $P$ be as given in the hypothesis. For the purpose of obtaining a contradiction, assume that $P \in B P(C)$. Then there exists $x^{\prime} \in X$ where $\phi\left(x^{\prime}\right)_{B_{k}}=C$ and $P=x_{\partial\left(B_{k}\right)}^{\prime}$.

Consider the configuration $z$ on $\mathbb{Z}^{d}$ that agrees with $x^{\prime}$ on $B_{k}$ and with $x$ on $F_{k}$. Note that $z$ agrees with both $x$ and $x^{\prime}$ on $\partial\left(B_{k}\right)$, because $x_{\partial\left(B_{k}\right)}=x_{\partial\left(B_{k}\right)}^{\prime}=P$. As a result (and because $\left(X, \mathbb{Z}^{d}\right)$ is a matrix shift), $z \in X$.

Therefore $\phi(z) \in Y$ and $\phi(z)_{F_{k}} \in E_{Y}\left(\phi(z)_{B_{k}}\right)$. By the definition of $z$,

$$
\phi(z)_{F_{k}}=\phi\left(z_{F_{k}}\right)=\phi\left(x_{F_{k}}\right)=\phi(x)_{F_{k}}=y_{F_{k}}
$$

and

$$
\phi(z)_{B_{k}}=\phi\left(z_{B_{k}}\right)=\phi\left(x_{B_{k}}^{\prime}\right)=\phi\left(x^{\prime}\right)_{B_{k}}=C,
$$

and we have $y_{F_{k}} \in E_{Y}(C)$, which is a contradiction. The assumption that $P \in$ $B P(C)$ must be false, as desired.

Theorem 2.3. Let $\left(Y, \mathbb{Z}^{d}\right)$ be a shift space. Suppose given any $M>0$, there exists $a k>0$, an $m>M^{k^{d-1}}$, and occurring configurations $\left\{C_{i}\right\}_{i=1}^{m}$ on $B_{k}$ for which $\left\{E_{Y}\left(C_{i}\right)\right\}_{i=1}^{m}$ is a union-increasing sequence. Then $\left(Y, \mathbb{Z}^{d}\right)$ is non-sofic.

Proof. Assume the hypothesis, but suppose contrary to the conclusion that there exists a matrix shift $\left(X, \mathbb{Z}^{d}\right)$ and a 1-block code $\phi: X \rightarrow Y$.

Let $\mathcal{A}$ be the alphabet for $X$, let $M=|\mathcal{A}|^{2 d}$, and choose $k>0, m>M^{k^{d-1}}$, and occurring configurations $\left\{C_{i}\right\}_{i=1}^{m}$ on $B_{k}$ for which $\left\{E_{Y}\left(C_{i}\right)\right\}_{i=1}^{m}$ is a union-increasing sequence.

For each $1 \leq i \leq m$, choose $y^{(i)} \in Y$ so that $y_{B_{k}}^{(i)}=C_{i}$ and $y_{F_{k}}^{(i)} \notin \bigcup_{j<i} E_{Y}\left(C_{j}\right)$. This is possible because $\left\{E_{Y}\left(C_{i}\right)\right\}_{i=1}^{m}$ is a union-increasing sequence.

For each $i$, let $x^{(i)} \in X$ be some pre-image of $y^{(i)}$ and write $P_{i}=x_{\partial\left(B_{k}\right)}^{(i)}$. Note that $P_{i} \in B P\left(C_{i}\right)$. For $j<i$, however, Lemma 2.2 implies that $P_{i} \notin B P\left(C_{j}\right)$, and therefore $P_{i} \neq P_{j}$. This implies that the $m$ configurations $P_{i}$ are distinct.

On the other hand, there are fewer than $2 d \cdot k^{d-1}$ lattice points in $\partial\left(B_{k}\right)$; hence there are fewer than $|\mathcal{A}|^{2 d \cdot k^{d-1}}=M^{k^{d-1}}$ distinct configurations on $\partial\left(B_{k}\right)$ using symbols from $\mathcal{A}$.

This yields a contradiction, because $m$ was chosen to be greater than $M^{k^{d-1}}$, and thus $\left(Y, \mathbb{Z}^{d}\right)$ is non-sofic.

We conjecture that if $\left(X, \mathbb{Z}^{d}\right)$ is sofic, then $\log \left(\left|\mathcal{E}_{X}^{k}\right|\right)$ must grow more slowly than $k^{d}$; that is, that $\log \left(\left|\mathcal{E}_{X}^{k}\right|\right) \in o\left(k^{d}\right)$. For $d>1$, the converse of this conjecture is certainly not true. To see this, first note that the number of occurring configurations on $B_{k}$ is an upper bound for $\left|\mathcal{E}_{Y}^{k}\right|$. Take $\left(Y, \mathbb{Z}^{d}\right)$ to be any non-sofic shift space for 
which the number of occurring configurations on $B_{k}$ is less than $M^{k^{d-1}}$ for some $M>0$ and for all $k \in \mathbb{N}$. One way to create such an example is to let $\left(X, \mathbb{Z}^{d-1}\right)$ be a non-empty shift space whose set of forbidden configurations is not recursively enumerable. Then create $\left(Y, \mathbb{Z}^{d}\right)$, where points in $Y$ are constructed by "stacking" copies of the same point from $X$ in the $d$-direction. That is,

$$
Y=\left\{y \mid y_{\left\{\vec{v} \in \mathbb{Z}^{d} \mid v_{d}=0\right\}}=x \text { for some } x \in X \text { and } \sigma_{\vec{e}_{d}}(y)=y\right\} .
$$

Such counterexamples have zero entropy; we know of no positive entropy counterexamples to the converse of the conjecture.

We close this section with three non-sofic examples. In Example 2.4 the extender sets used in Theorem 2.3 are disjoint. This is not the case for Example 2.5. Example 2.6 is a one-dimensional example (that is known to be non-sofic because $\mathcal{R}_{Y}$ is not finite) for which there do not exist configurations $\left\{C_{i}\right\}$ satisfying the hypotheses of Theorem 2.3. This example shows that Theorem 2.3 does not completely characterize non-soficness. Example 2.6 was first described in $[\mathrm{P}]$.

Example 2.4. Let $\mathcal{A}=\{F, G, 0,1\}$ and let $d>1$. Define a collection of configurations $\mathcal{W}$ on $B_{k}^{d-1} \subset \mathbb{Z}^{d-1}$ via

$$
W \in \mathcal{W} \text { iff } W_{\vec{v}}=\left\{\begin{array}{l}
F \text { for } \vec{v} \in \partial\left(B_{k}^{d-1}\right), \\
G \text { for } \vec{v} \in B_{k}^{d-1} \backslash \partial\left(B_{k}^{d-1}\right) .
\end{array}\right.
$$

Intuitively the configurations $W \in \mathcal{W}$ are "windows", and the symbols $F$ and $G$ stand for frame and glass respectively. Let $D_{k} \subset \mathbb{Z}^{d}$ be defined as

$$
D_{k}=B_{k}^{d-1} \times[-k, k]=\left(B_{k}^{d-1} \times[-k,-1]\right) \cup\left(B_{k}^{d-1} \times\{0\}\right) \cup B_{k}^{d} .
$$

Define $Y \subset \mathcal{A}^{\mathbb{Z}^{d}}$ via forbidden blocks

$$
\mathcal{F}=\left\{C \in \mathcal{A}^{D_{k}} \mid C_{B_{k}^{d-1} \times\{0\}} \in \mathcal{W} \text { and } C_{B_{k}^{d}} \neq C_{B_{k}^{d-1} \times[-k,-1]}\right\} .
$$

Then $\left(Y, \mathbb{Z}^{d}\right)$ is non-sofic.

Proof. Let $M>0$. Choose $k$ so that $2^{k-1}>M$, and note that this implies that $2^{(k-1) k^{d-1}}>M^{k^{d-1}}$. Let $m=2^{(k-1) k^{d-1}}$, and note that there are $m$ distinct configurations of 0 's and 1's on $B_{k}^{d-1} \times[2, k]$. Extend each of these $m$ configurations to a configuration on $B_{k}^{d}$ by placing a window at $B_{k}^{d-1} \times\{1\}$. Let $\left\{C_{i}\right\}$ be the resulting set of configurations on $B_{k}^{d}$. These configurations contain no forbidden blocks; hence they occur and their extender sets $E_{Y}\left(C_{i}\right)$ are non-empty.

By the construction of the $C_{i}$, every configuration $y \in E_{Y}\left(C_{i}\right)$ contains an "imprint" of $C_{i}$. More precisely, $y_{B_{k}^{d-1} \times[-k+2,0]}$ matches the configuration of 0's and 1's within $C_{i}$. (Otherwise, extending $C_{i}$ by $y$ would create a forbidden block.) As a result, $y$ cannot extend $C_{j}$ for $j \neq i$, because distinct $C_{i}$ contain distinct configurations of 0 's and 1's.

The $\left\{E_{Y}\left(C_{i}\right)\right\}_{i=1}^{m}$ are then distinct non-empty sets which always form a unionincreasing sequence, and by Theorem $2.3,\left(Y, \mathbb{Z}^{d}\right)$ is non-sofic.

Example 2.5. Let $\mathcal{A}=\{0,1,2\}$. Define $Y \subset \mathcal{A}^{\mathbb{Z}^{2}}$ via the following set of forbidden configurations:

$$
\mathcal{F}=\left\{C \in \mathcal{A}^{B_{k}} \mid k \in \mathbb{N} \text { and } C_{\vec{v}}=\left\{\begin{array}{ll}
1 & \text { for } \vec{v} \in[1, k] \times\{1\} \\
2 & \text { for } \vec{v} \in[1, k] \times\{k\}
\end{array}\right\} .\right.
$$


Intuitively $\mathcal{F}$ includes all configurations on $B_{k}$ in which the first row of $B_{k}$ consists entirely of 1 's and the $k^{\text {th }}$ row of $B_{k}$ consists entirely of 2 's. Then $\left(Y, \mathbb{Z}^{2}\right)$ is nonsofic.

Proof. Let $M>0$ be given. Choose $k=M+1$ and let $m=(M+1)^{M+1}=k^{k}$. We will show that there exists $\left\{y^{(i)}\right\}_{i=1}^{m} \subset Y$ with

$$
y_{F_{k}}^{(i)} \notin E_{Y}\left(y_{B_{k}}^{(j)}\right) \text { for all } 1 \leq j<i \leq m .
$$

Then, for $C_{i}=y_{B_{k}}^{(i)}$ for $1 \leq i \leq m$, the set $\left\{E_{Y}\left(C_{i}\right)\right\}_{i=1}^{m}$ is union-increasing, and thus it will follow from Theorem 2.3 that $\left(Y, \mathbb{Z}^{2}\right)$ is non-sofic.

For $1 \leq i \leq m$, let $\vec{a}_{i}=\left(a_{i}^{1}, a_{i}^{2}, \ldots, a_{i}^{k}\right)$ be the $i^{t h} k$-tuple in reverse lexicographic order among all $k$-tuples with integer entries between 1 and $k$ inclusive. That is, if $1 \leq i<j \leq m$, then $\vec{a}_{i}$ comes after $\vec{a}_{j}$ in lexicographic order so that if $1 \leq J \leq k$ is the first index for which $a_{i}^{J} \neq a_{j}^{J}$, then $a_{i}^{J}>a_{j}^{J}$.

We will first describe the points $y^{(i)} \in Y$ informally. On the $j^{\text {th }}$ row of $y^{(i)}$, for $1 \leq j \leq k$, there will be a word of length $k+2(k-j)$ consisting entirely of 1 's with the last $a_{i}^{j}$ of those 1's intersecting the left side of $B_{k}$. Then, for each $1 \leq j \leq k$, corresponding to the word consisting entirely of 1 's on the $j^{\text {th }}$ row as described there will be a word of length $k+2(k-j)+1$ (exactly one unit longer than the word of 1 's) consisting entirely of 2's on the $(3 k-j)^{t h}$ row of $y^{(i)}$. This word consisting of 2's will be located with its leftmost 2 in the same column as the leftmost 1 of the corresponding word consisting of 1 's and with its last $a_{i}^{j}+12$ 's on the left side of $B_{3 k}$. Elsewhere, $y^{(i)}$ will contain 0's.

Figure 1 illustrates $y^{(i)}$ for $k=5$ and $\vec{a}_{i}=(5,3,4,0,5)$.

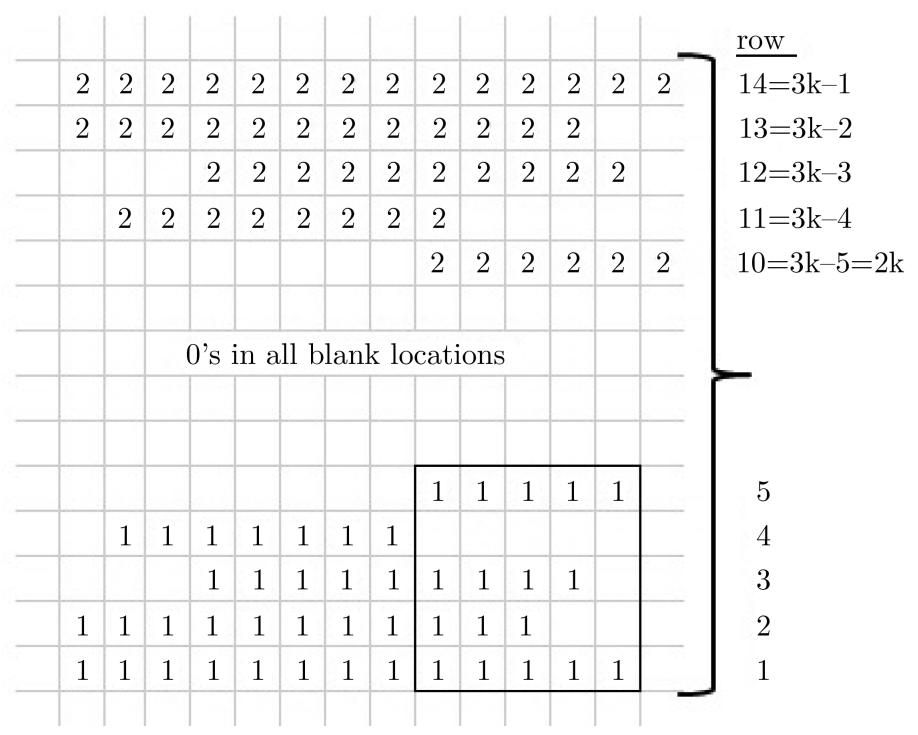

$$
\begin{aligned}
& \frac{\text { number of } 2 \text { 's }}{14=\mathrm{k}+2(\mathrm{k}-1)+1} \\
& 12=\mathrm{k}+2(\mathrm{k}-2)+1 \\
& 10=\mathrm{k}+2(\mathrm{k}-3)+1 \\
& 8=\mathrm{k}+2(\mathrm{k}-4)+1 \\
& 6=\mathrm{k}+2(\mathrm{k}-5)+1
\end{aligned}
$$

number of 1's

$5=\mathrm{k}+2(\mathrm{k}-5)$

$7=\mathrm{k}+2(\mathrm{k}-4)$

$9=\mathrm{k}+2(\mathrm{k}-3)$

$11=\mathrm{k}+2(\mathrm{k}-2)$

$13=\mathrm{k}+2(\mathrm{k}-1)$

Figure 1. Example showing $y^{(i)}$ for $k=5$ and $\vec{a}_{i}=(5,3,4,0,5)$, with $B_{k}$ outlined. 
More formally, define $y^{(i)}$ as follows:

(1) $y_{(\ell, j)}^{(i)}=1$ for $1 \leq j \leq k$ and $-k-2(k-j)+a_{i}^{j}-1 \leq \ell \leq a_{i}^{j}$;

(2) $y_{(\ell, 3 k-j)}^{(i)}=2$ for $1 \leq j \leq k$ and $-k-2(k-j)+a_{i}^{j}-1 \leq \ell \leq a_{i}^{j}+1$;

(3) $y_{\vec{v}}^{(i)}=0$ elsewhere.

We leave it to the reader to verify that the points $\left\{y^{(i)}\right\}_{i=1}^{m}$ are in $Y$ and that $y_{F_{k}}^{(i)} \notin E_{Y}\left(y_{B_{k}}^{(j)}\right)$ for all $1 \leq j<i \leq m$.

Example 2.6 $(\underline{\mathrm{P}})$. Let $(Y, \mathbb{Z})$ be the one-dimensional reverse context-free shift on the alphabet $\mathcal{A}=\{a, b, c\}$ given by forbidden blocks

$$
\mathcal{F}=\left\{c a^{m} b^{m} c \mid m>0\right\} .
$$

Then $(Y, \mathbb{Z})$ is non-sofic, but there do not exist configurations whose extender sets satisfy the hypotheses of Theorem 2.3 .

A number of observations will be useful in the proof of this claim. The first follows immediately from the definition of $\mathcal{F}$ :

Observation 2.7. Let $w$ be a word occurring in the reverse context-free shift space $Y$. Then:

(1) For $i \geq 0, w b^{i} c$ (respectively $c a^{i} w$ ) is forbidden if and only if $w$ ends with $c a^{i+j} b^{j}$ (begins with $a^{j} b^{i+j} c$ ) with $j \geq 0$ and $i+j>0$;

(2) For $0<i \leq j, w a^{i} b^{j} c\left(c a^{j} b^{i} w\right)$ is forbidden if and only if $w$ ends with $c a^{j-i}$ (begins with $b^{j-i} c$ ).

The following two observations are simple consequences of Observation 2.7

Observation 2.8. Let $w$ be a word occurring in the reverse context-free shift space $Y$. Then $w b^{i} c$ and $w b^{j} c$ (or $c a^{i} w$ and $c a^{j} w$ ) are both forbidden if and only if $j=i$.

Proof. By Observation 2.7, $w b^{i} c$ is forbidden if and only if $w$ ends with $c a^{i+k} b^{k}$ for some $k \geq 0$. Again using Observation 2.7

$$
w b^{j} c=w^{\prime} c a^{i+k} b^{k} b^{j} c
$$

is forbidden if and only if $i+k=j+k$ or $i=j$, as desired.

Observation 2.9. Let $w$ be a word occurring in the reverse context-free shift space $Y$. Then $w a^{i} b^{j} c$ and $w a^{\ell} b^{m} c$ (or $c a^{j} b^{i} w$ and $c a^{m} b^{\ell} w$ ), where $0<i \leq j$ and $0<\ell \leq m$, are both forbidden if and only if $j-i=m-\ell$.

Proof. By Observation 2.7 $w a^{i} b^{j} c$ is forbidden if and only if $w$ ends with $c a^{j-i}$. Again using Observation 2.7 .

$$
w a^{\ell} b^{m} c=w^{\prime} c a^{j-i} a^{\ell} b^{m} c
$$

is forbidden if and only if $j-i+\ell=m$ or $j-i=m-\ell$, as desired.

Let $k>0$ be given and let $\mathcal{W}$ be all occurring words of length $k$ in $Y$. In the proof of Claim 2.10, it will be helpful to partition $\mathcal{W}$ into twelve subsets. The first three subsets are $\mathcal{W}_{a}=\left\{a^{k}\right\}, \mathcal{W}_{b}=\left\{b^{k}\right\}$, and $\mathcal{W}_{a b}=\left\{a^{i} b^{k-i}\right.$ for $\left.1<i<k\right\}$.

The next eight subsets include all the words $w$ that either begin with $a^{i} b^{j} c$ for $j \geq 0$ and $i \leq j$ or end with $c a^{j} b^{i}$ for $j \geq 0$ and $i \leq j$ or both. These subsets can be indexed by elements in

$$
(\mathcal{L} \times \mathcal{R}) \backslash\{(0,0)\}=(\{b c, a b c, 0\} \times\{c a, c a b, 0\}) \backslash\{(0,0)\} .
$$


The element from $\mathcal{L}$ indicates (respectively) whether $w$ begins with $b^{j} c$ for $j \geq 0$, with $a^{i} b^{j} c$ for $0<i \leq j$, or neither. Similarly, the element from $\mathcal{R}$ indicates whether $w$ ends with $c a^{j}$ for $j \geq 0$, with $c a^{j} b^{i}$ for $0<i \leq j$, or neither. For example, $\mathcal{W}_{(b c, 0)}$ consists of all words of length $k$ of the form $b^{\ell} c w^{\prime}$ for some $\ell \geq 0$ where $w^{\prime}$ does not end in $c a^{j} b^{i}$ for $j \geq 0$ and $i \leq j$.

Let the twelfth subset of $\mathcal{W}$, denoted $\mathcal{W}_{\text {free }}$, be all the elements of $\mathcal{W}$ that do not fall into one of the previously defined subsets. (These are all the occurring words of length $k$ with the full extender set $\left\{y_{F_{k}} \mid y \in Y\right\}$.)

We are now ready to prove Claim 2.10

Claim 2.10. Let $(Y, \mathbb{Z})$ be the reverse context-free shift. Let $n>84, k>0$, and suppose that $\left\{C_{i}\right\}_{i=1}^{n}$ is a collection of occurring configurations on $B_{k}$. Then $\left\{E_{Y}\left(C_{i}\right)\right\}_{i=1}^{n}$ is not a union-increasing sequence.

Proof. Suppose that $\left\{C_{i}\right\}_{i=1}^{n}$ is a collection of occurring configurations on $B_{k}$. We will show that $\left\{E_{Y}\left(C_{i}\right)\right\}_{i=1}^{n}$ is not a union-increasing sequence.

The set $\left\{C_{i}\right\}_{i=1}^{n}$ can be partitioned into twelve different sets as previously described. By the pigeonhole principle, at least one of those sets has more than seven elements. Let $\left\{C_{i_{j}}\right\}_{j=1}^{m} \subseteq\left\{C_{i}\right\}_{i=1}^{n}, m>7$, be such that $\left\{C_{i_{j}}\right\}_{j=1}^{m}$ are all in the same partition subset. In order to simplify notation in what follows, without loss of generality we can relabel these $C_{i_{j}}$ in a way that preserves the original ordering as $\left\{C_{i}\right\}_{i=1}^{m}$.

There are twelve cases to check, depending on the partition element containing the points $\left\{C_{i}\right\}_{i=1}^{m}$. We check one case in order to illustrate the nature of the arguments involved and leave the remaining details to the reader.

Suppose $\left\{C_{i}\right\}_{i=1}^{m} \subseteq \mathcal{W}_{(a b c, c a b)}$. In this case, for each $1 \leq i \leq m$,

$$
C_{i}=a^{\ell_{i}} b^{\ell_{i}^{\prime}} c \cdots c a^{r_{i}^{\prime}} b^{r_{i}}
$$

for $0<\ell_{i} \leq \ell_{i}^{\prime}$ and $0<r_{i} \leq r_{i}^{\prime}$. We will show that $\left\{E_{Y}\left(C_{i}\right)\right\}_{i=1}^{m}$ is not union increasing.

Because $C_{m}$ occurs in $Y$, clearly there are points $y \in Y$ with $y_{B_{k}}=C_{m}$. If for each of these points there exists $1 \leq i<m$ for which $y_{F_{k}} \in E_{Y}\left(C_{i}\right)$, we are done, since in this case $E_{Y}\left(C_{m}\right) \subseteq \bigcup_{i=1}^{m-1} E_{Y}\left(C_{i}\right)$ and $\left\{E\left(C_{i}\right)\right\}$ is not union-increasing. Thus we may choose $y^{(m)} \in Y$ so that $y_{B_{k}}^{(m)}=C_{m}$ and $y_{F_{k}}^{(m)} \notin E_{Y}\left(C_{i}\right)$ for all $1 \leq i<m$.

So either $y_{(-\infty, 0]}^{(m)} C_{i}$ is forbidden or $C_{i} y_{[k+1, \infty)}^{(m)}$ is forbidden (or both) for each $1 \leq i<m$. We will assume $y_{(-\infty, 0]}^{(m)} C_{i}$ is forbidden for at least half of the $i$ 's. (If this is not the case, then $C_{i} y_{[k+1, \infty)}^{(m)}$ is forbidden for at least half of the $i$ 's, and a similar argument holds.) Using Observation 2.9. for these $i$ 's we have $\ell_{i}^{\prime}-\ell_{i}=\ell_{m}^{\prime}-\ell_{m}$. Again, without loss of generality, by relabeling in a way that preserves order, we may let $\left\{C_{i}\right\}_{i=1}^{p}$ be such that $\ell_{i}^{\prime}-\ell_{i}=\ell_{m}^{\prime}-\ell_{m}$ for all $1 \leq i \leq p$ with $p>3$.

Because $C_{p}$ occurs in $Y$, clearly there are points $y \in Y$ with $y_{B_{k}}=C_{p}$. If for each of these points there exists $1 \leq i<p$ for which $y_{F_{k}} \in E_{Y}\left(C_{i}\right)$, we are done, since in this case $E_{Y}\left(C_{p}\right) \subseteq \bigcup_{i=1}^{p-1} E_{Y}\left(C_{i}\right)$ and $\left\{E\left(C_{i}\right)\right\}$ is not union-increasing. Thus we may choose $y^{(p)} \in Y$ so that $y_{B_{k}}^{(p)}=C_{p}$ and $y_{F_{k}}^{(p)} \notin E_{Y}\left(C_{i}\right)$ for all $1 \leq i<p$. 
Because $\ell_{i}^{\prime}-\ell_{i}=\ell_{m}^{\prime}-\ell_{m}$ for $1 \leq i \leq p$, by Observation 2.9] $y_{(\infty, 0]}^{(p)} C_{i}$ cannot be forbidden for any $1 \leq i<p$. Thus we must have $C_{i} y_{[k+1, \infty)}^{(p)}$ forbidden for each $1 \leq i<p$. But then, by Observation 2.9, we must have $r_{i}^{\prime}-r_{i}=r_{p}^{\prime}-r_{p}$ for all $1 \leq i<p$. In particular, this holds for $i=1$ and $i=2$. Thus, because

$$
\ell_{1}^{\prime}-\ell_{1}=\ell_{2}^{\prime}-\ell_{2}=\ell_{m}^{\prime}-\ell_{m} \text { and } r_{1}^{\prime}-r_{1}=r_{2}^{\prime}-r_{2}=r_{p}^{\prime}-r_{p},
$$

we must have $E_{Y}\left(C_{1}\right)=E_{Y}\left(C_{2}\right)$, and thus $\left\{E_{Y}\left(C_{i}\right)\right\}_{i=1}^{n}$ is not a union-increasing sequence, as desired.

\section{Soficness AND SUbShifTS OF The FORM $\left(Y^{\mathbb{Z}}, \mathbb{Z}^{d}\right)$}

Let $\left(Y, \mathbb{Z}^{d}\right)$ be a shift space. For

$$
\pi(Y)=\left\{y_{\mathbb{Z} \times \vec{v}} \mid y \in Y, \vec{v} \in \mathbb{Z}^{d-1}\right\},
$$

the subshift $(\pi(Y), \mathbb{Z})$ is called the $\vec{e}_{1}$-projection of $\left(Y, \mathbb{Z}^{d}\right)$. Properties of projectional dynamical systems have been studied in [JKM], [PS], and [S].

Let $\left(Y, \mathbb{Z}^{d-1}\right)$ be a shift space and let $Y^{\mathbb{Z}}$ be the usual infinite Cartesian product of $Y$. It is easy to see that $\left(Y^{\mathbb{Z}}, \mathbb{Z}^{d}\right)$ is a shift space.

Note that if $\left(Y, \mathbb{Z}^{d-1}\right)$ is a $(d-1)$-dimensional shift space, then $Y$ and $\pi\left(Y^{\mathbb{Z}}\right)$ are the same. On the other hand, for a $d$-dimensional shift space $\left(Y, \mathbb{Z}^{d}\right), Y \subseteq(\pi(Y))^{\mathbb{Z}}$, but $(\pi(Y))^{\mathbb{Z}}$ and $Y$ may or may not be the same.

In this section we explore the relationship between the soficness of $\left(Y, \mathbb{Z}^{d-1}\right)$ and of $\left(Y^{\mathbb{Z}}, \mathbb{Z}^{d}\right)$. It is not difficult to show that if $\left(Y, \mathbb{Z}^{d-1}\right)$ is sofic, then $\left(Y^{\mathbb{Z}}, \mathbb{Z}^{d}\right)$ is sofic as well:

Observation 3.1. Let $\left(Y, \mathbb{Z}^{d-1}\right)$ be sofic. Then $\left(Y^{\mathbb{Z}}, \mathbb{Z}^{d}\right)$ is sofic.

Proof. Because $\left(Y, \mathbb{Z}^{d-1}\right)$ is sofic, $\phi: X \rightarrow Y$ for some matrix shift $\left(X, \mathbb{Z}^{d-1}\right)$ where $\phi$ is a 1-block code. Let $\mathcal{A}$ be the alphabet for $X$.

The subshift $\left(X^{\mathbb{Z}}, \mathbb{Z}^{d}\right)$ is also a matrix shift with alphabet $\mathcal{A}$. (The $\vec{e}_{i}$-transition rules, $1 \leq i \leq d-1$, for $\left(X^{\mathbb{Z}}, \mathbb{Z}^{d}\right)$ are the $\vec{e}_{i}$-transition rules for $\left(X, \mathbb{Z}^{d-1}\right)$, and all $\vec{e}_{d}$-transitions are allowed.) With $\phi$ extended to $X^{\mathbb{Z}}$ in the obvious way, we have $\phi: X^{\mathbb{Z}} \rightarrow Y^{\mathbb{Z}}$. Thus, $\left(Y^{\mathbb{Z}}, \mathbb{Z}^{d}\right)$ is sofic.

The converse to Observation 3.1 was first stated as a conjecture by E. Jeandel at Math-Info 2010 in Marseille P2.

Conjecture 3.2. Let $\left(Y, \mathbb{Z}^{d-1}\right)$ be non-sofic. Then $\left(Y^{\mathbb{Z}}, \mathbb{Z}^{d}\right)$ is non-sofic.

This conjecture remains open; however, in this section, we will prove a partial converse to Observation 3.1 Theorem 3.3 says that if a subshift $\left(Y, \mathbb{Z}^{d-1}\right)$ satisfies the hypotheses of Theorem 2.3 , then the free product $\left(Y^{\mathbb{Z}}, \mathbb{Z}^{d}\right)$ also satisfies those hypotheses and thus is non-sofic.

Theorem 3.3. For $d \geq 2$, let $\left(Y, \mathbb{Z}^{d-1}\right)$ be a $(d-1)$-dimensional shift space that satisfies the hypotheses of Theorem 2.3 . Then $\left(Y^{\mathbb{Z}}, \mathbb{Z}^{d}\right)$ also satisfies the hypotheses of Theorem 2.3 (and thus is non-sofic).

Proof. Suppose that $\left(Y, \mathbb{Z}^{d-1}\right)$ satisfies the hypotheses of Theorem 2.3. Let $M>0$ be given and, as described in Theorem 2.3, choose $k>0, m>M^{k^{d-2}}$ and a collection of occurring configurations $\mathcal{C}=\left\{C_{i}\right\}_{i=1}^{m}$ on $B_{k}^{d-1} \subset \mathbb{Z}^{d-1}$ for which $\left\{E_{Y}\left(C_{i}\right)\right\}_{i=1}^{m}$ is a union-increasing sequence. 
Because $\left\{E_{Y}\left(C_{i}\right)\right\}_{i=1}^{m}$ is a union-increasing sequence, we may choose points $\left\{x^{(i)}\right\}_{i=1}^{m} \subseteq Y$ with $x_{B_{k}^{d-1}}^{(i)}=C_{i}$ and

$$
x_{F_{k}^{d-1}}^{(i)} \notin E_{Y}\left(C_{j}\right) \text { whenever } 1 \leq j<i \leq m .
$$

We will use this collection of points in $Y$ to create a collection of points in $Y^{\mathbb{Z}}$ (and from these a collection of occurring configurations on $B_{k}^{d}$ ) as follows:

For $1 \leq i \leq m^{k}$, let $\vec{a}_{i}=\left(a_{i}^{1}, a_{i}^{2}, \ldots, a_{i}^{k}\right)$ be the $i^{\text {th }} k$-tuple in lexicographic order among all $k$-tuples with integer entries between 1 and $m$ inclusive. For each $\vec{a}_{i}$, create $y^{(i)} \in Y^{\mathbb{Z}}$ with

$$
y_{\mathbb{Z}^{d-1}}^{(i)} \times\{1,2, \ldots, k\}=x^{\left(a_{i}^{1}\right)} \times x^{\left(a_{i}^{2}\right)} \times \ldots \times x^{\left(a_{i}^{k}\right)} .
$$

Because $Y^{\mathbb{Z}}$ is a free product, such points clearly exist.

Consider the collection of configurations $\left\{y_{B_{k}^{d}}^{(i)}\right\}_{i=1}^{m^{k}}$ on $B_{k}^{d} \subset \mathbb{Z}^{d}$ which occur in $Y^{\mathbb{Z}}$. Clearly

$$
y_{B_{k}^{d}}^{(i)}=C_{a_{i}^{1}} \times C_{a_{i}^{2}} \times \ldots \times C_{a_{i}^{k}} .
$$

Then the set $\left\{E_{Y^{\mathbb{Z}}}\left(y_{B_{k}^{d}}^{(i)}\right)\right\}_{i=1}^{m^{k}}$ is union-increasing. To see this, let $1 \leq j<i \leq m^{k}$. Then $\vec{a}_{j}$ is less than $\vec{a}_{i}$ in lexicographic order, and we may choose $1 \leq J \leq k$ to be the smallest index for which $a_{j}^{J} \neq a_{i}^{J}$ (and $a_{j}^{J}<a_{i}^{J}$ ). But then

$$
x_{F_{k}^{d-1}}^{\left(a_{i}^{J}\right)} \notin E_{Y}\left(C_{a_{j}^{J}}\right) \text { and hence } y_{F_{k}^{d}}^{(i)} \notin E_{Y^{\mathbb{Z}}}\left(y_{B_{k}^{d}}^{(j)}\right) .
$$

Thus, $k>0, m^{k}>\left(M^{k^{d-2}}\right)^{k}=M^{k^{d-1}}$ and the collection of occurring configurations $\left\{y_{B_{k}^{d}}^{(i)}\right\}_{i=1}^{m^{k}}$ in $Y^{\mathbb{Z}}$ on $B_{k}^{d} \subset \mathbb{Z}^{d}$ satisfy the hypotheses of Theorem 2.3, as desired.

We end this section with two examples. In the first example, we apply Theorem 3.3 to the one-dimensional context-free shift, showing that its free product is non-sofic. In the second example, we do the same thing for a variation on the one-dimensional context-free shift.

Example 3.4. Let $(Y, \mathbb{Z})$ be the one-dimensional context-free shift on the alphabet $\mathcal{A}=\{a, b, c\}$ given by forbidden blocks

$$
\mathcal{F}=\left\{c a^{n} b^{m} c, \text { where } n \neq m\right\} .
$$

Then $\left(Y^{\mathbb{Z}^{d-1}}, \mathbb{Z}^{d}\right)$ is non-sofic for all $d \geq 1$.

Proof. We will show that $(Y, \mathbb{Z})$ satisfies the hypotheses of Theorem 2.3. Repeated applications of Theorem 3.3 then establish the claim.

Let $M>0$ be given and choose $k$ large enough that $m=(k-2)>M$. Consider the occurring words of length $k$ in $(Y, \mathbb{Z})$ of the form

$$
C_{i}=c^{k-1-i} a^{i} b, \text { where } 1 \leq i \leq k-2 .
$$

Note that $C_{i}$ can only be followed by a word of the form $b^{j} c$ if $j=i-1$. Let $U_{i}=b^{i-1} c$.

For each $1 \leq i \leq m$ consider $y^{(i)} \in Y$ with $y_{B_{k}}^{(i)}=C_{i}$, where

$$
y^{(i)}=\cdots \operatorname{cccc} . C_{i} U_{i} \operatorname{ccccc} \cdot \cdots \text {. }
$$


Clearly $y_{F_{k}}^{(i)} \notin E_{Y}\left(C_{j}\right)$ for all $i \neq j$. So $\left\{E_{Y}\left(C_{i}\right)\right\}_{i=1}^{m}$ is union-increasing and $(Y, \mathbb{Z})$ satisfies the hypotheses of Theorem 2.3 , as desired.

Example 3.5. Let $(Y, \mathbb{Z})$ be the one-dimensional modified context-free shift on the alphabet $\mathcal{A}=\{a, b, c\}$ given by forbidden blocks

$$
\mathcal{F}=\left\{c a^{n} b^{m} c, \text { where } n<m\right\} .
$$

Then $\left(Y^{\mathbb{Z}^{d-1}}, \mathbb{Z}^{d}\right)$ is non-sofic for all $d \geq 1$.

Proof. Let $M>0$ be given, and let $k, m,\left\{C_{i}\right\}_{i=1}^{m}$ and $\left\{y^{(i)}\right\}_{i=1}^{m}$ be as described in the proof found in Example 3.4. Then clearly $y_{F_{k}}^{(i)} \in E_{Y}\left(C_{i}\right)$, but $y_{F_{k}}^{(i)} \notin E_{Y}\left(C_{j}\right)$ for all $j<i$. Thus $\left\{E_{Y}\left(C_{i}\right)\right\}_{i=1}^{m}$ is union-increasing, and by repeated applications of Theorem 3.3 . $\left(Y^{\mathbb{Z}^{d-1}}, \mathbb{Z}^{d}\right)$ is non-sofic, as desired.

Note that it remains an open question whether or not $\left(Y^{\mathbb{Z}^{d-1}}, \mathbb{Z}^{d}\right), d \geq 2$, is sofic when $(Y, \mathbb{Z})$ is the reverse context-free shift (Example 2.6).

\section{Further observations}

4.1. An alternative partial converse to Observation 3.1. In Observation 4.1, we provide a partial converse to Observation 3.1 that does not rely on Theorem 2.3. Together with Theorem 3.3, this provides information on the nature of a counterexample to Conjecture 3.2, should one exist.

Observation 4.1. Let $\left(Y, \mathbb{Z}^{d-1}\right)$ be a shift space. Suppose that $\phi: X \rightarrow Y^{\mathbb{Z}}$ is a 1-block code where $\left(X, \mathbb{Z}^{d}\right)$ is a matrix shift given by matrices $H_{1}, H_{2}, \ldots, H_{d}$. Suppose any word allowed by $H_{1}, H_{2}, \ldots, H_{d-1}$ occurs in $X$. Then $\left(Y, \mathbb{Z}^{d-1}\right)$ is sofic.

Proof. Let $\mathcal{A}$ be the alphabet of $X$ and let $\left(X^{\prime}, \mathbb{Z}^{d-1}\right)$ be the $(d-1)$-dimensional matrix shift with alphabet $\mathcal{A}$ and transition rules given by $H_{1}, H_{2}, \ldots, H_{d-1}$. We'd like to show that $\phi: X^{\prime} \rightarrow Y$.

Points in $X$ must satisfy the transition rules given by $H_{1}, H_{2}, \ldots, H_{d}$, which implies that $\pi(X) \subseteq X^{\prime}$ where $\pi(X)=\left\{x_{\mathbb{Z}^{d-1} \times\{0\}} \mid x \in X\right\}$. In addition, the assumption that all words allowed by $H_{1}, H_{2}, \ldots, H_{d-1}$ occur in $X$ implies that $X^{\prime} \subseteq \pi(X)$ as well.

Taken together, $\pi(X) \subseteq X^{\prime}$ and $X^{\prime} \subseteq \pi(X)$ give $X^{\prime}=\pi(X)$, but then

$$
\phi\left(X^{\prime}\right)=\phi(\pi(X))=\pi(\phi(X))=\pi\left(Y^{\mathbb{Z}}\right)=Y,
$$

as desired.

4.2. Synchronizing words and non-soficness. Here we identify a class of onedimensional shifts $(Y, \mathbb{Z})$ that satisfy the hypotheses of Theorem 2.3 . We note that by Theorem 3.3, this implies $\left(Y^{\mathbb{Z}^{d-1}}, \mathbb{Z}^{d}\right)$ is non-sofic for all $d \geq 1$. We begin with a definition.

Definition 4.2. Let $(Y, \mathbb{Z})$ be a shift space. A word $w$ occurring in $Y$ is said to be a synchronizing word if any time $u w$ and $w v$ occur in $Y$ for words $u$ and $v$, then $u w v$ occurs in $Y$ as well.

Observation 4.3. Let $(Y, \mathbb{Z})$ be a shift space that contains no synchronizing word. Then the hypotheses of Theorem 2.3 are satisfied. 
Proof. Suppose $(Y, \mathbb{Z})$ is a shift space that contains no synchronizing word. Let $M>0$. We will construct a collection of occurring configurations $\left\{C_{i}\right\}_{i=1}^{M+1}$ whose extender sets form a union-increasing sequence.

To do this, first let $w_{1}$ be any finite word that occurs in $Y$. Inductively define a sequence of occurring words $\left\{w_{i}\right\}_{i=1}^{M+1}$ as follows. Because $w_{i}$ is not synchronizing, there exist words $u_{i}$ and $v_{i}$ for which $u_{i} w_{i}$ and $w_{i} v_{i}$ occur in $Y$, but $u_{i} w_{i} v_{i}$ does not occur in $Y$. Define $w_{i+1}=u_{i} w_{i}$.

Note that for each $1 \leq i<j \leq M+1, w_{i} v_{i}$ occurs in $Y$ but $w_{j} v_{i}$ does not. This is by construction when $j=i+1$. When $j>i+1$, it follows from the fact that it holds for $j=i+1$ and also from the fact that words that can follow $w_{j}$ can necessarily follow $w_{\ell}$ for any $\ell<j$ since, by construction, $w_{j}=w w_{\ell}$ for some word $w$.

Let $K$ be the length of $w_{M+1}$ (the longest $w_{i}$ ), and let $k>K$. Because $w_{i} v_{i}$ occurs in $Y$ for each $1 \leq i \leq M+1$, we can find points $\left\{y^{(i)}\right\} \subseteq Y$ with

$$
y_{B_{k}}^{(i)}=t_{i} w_{i} \text { for some word } t_{i}
$$

and

$$
y^{(i)}=\cdots \cdot t_{i} w_{i} v_{i} \cdots
$$

Note that the preceding paragraph tells us that $y_{F_{k}}^{(i)} \notin E_{Y}\left(y_{B_{k}}^{(j)}\right)$ whenever $j>i$.

Consider $\left\{C_{i}\right\}_{i=1}^{M+1}$ where the $C_{i}$ are the $y_{B_{k}}^{(i)}$ listed in reverse order; that is, $C_{i}=y_{B_{k}}^{(M+2-i)}$. Then $k>0, m=M+1$, and $\left\{C_{i}\right\}_{i=1}^{m}$ satisfy the hypotheses of Theorem 2.3.

Corollary 4.4. Let $(Y, \mathbb{Z})$ be a shift space that contains no synchronizing word. Then $\left(Y^{\mathbb{Z}^{d-1}}, \mathbb{Z}^{d}\right)$ is non-sofic for all $d \geq 1$.

Proof. This follows immediately from Observation 4.3 and Theorem 3.3

We note that while Observation 4.3 shows that the lack of synchronizing words in $(Y, \mathbb{Z})$ is a sufficient condition for the non-soficness of $\left(Y^{\mathbb{Z}^{d-1}}, \mathbb{Z}^{d}\right)$, Examples 3.4 and 3.5 show that it is not a necessary condition. For both of these examples, the word $w=c$ is synchronizing, and yet $\left(Y^{\mathbb{Z}^{d-1}}, \mathbb{Z}^{d}\right)$ is non-sofic for all $d \geq 1$.

4.3. An alternative characterization of non-soficness. R. Pavlov has given a sufficient condition for the non-soficness of $\left(Y, \mathbb{Z}^{d}\right)$ under certain assumptions (Theorem 1.1, $\mathrm{P}$ ); this result is stated below (for $d=2$ ) in Theorem 4.6. In this subsection we observe that this result is a consequence of Theorem [2.3. In order to simplify notation, we prove that assertion only for $d=2$. We will show that Theorem 4.6 is a consequence of Theorem 2.3 using a proof that is similar in structure to the one used to prove Theorem 3.3. We begin with a necessary definition.

Definition 4.5. A shift space $\left(Y, \mathbb{Z}^{2}\right)$ is said to be block-gluing in the $\vec{e}_{2}$ direction with gluing distance $g \in \mathbb{N}$ if, given any pair of occurring configurations $C_{1}$ and $C_{2}$ on horizontal strips $S_{1}, S_{2} \subset \mathbb{Z}^{2}$ :

$$
S_{1}=\{(a, b) \mid a \in \mathbb{Z} \text { and } n \leq b \leq m\} \text { and } S_{2}=\left\{(a, b) \mid a \in \mathbb{Z} \text { and } m+g^{\prime} \leq b \leq p\right\}
$$

with $g^{\prime} \geq g$, there is a point $y \in Y$ with $y_{S_{i}}=C_{i}$ for $i=1,2$. 
Theorem $4.6\left([\mathrm{P})\right.$. Let $\left(Y, \mathbb{Z}^{2}\right)$ be a shift space where $(1)\left(Y, \mathbb{Z}^{2}\right)$ is block gluing in the $\vec{e}_{2}$ direction with gluing distance $g$, and $(2)$ the projection $(\pi(Y), \mathbb{Z})$ has no synchronizing word. Then $\left(Y, \mathbb{Z}^{2}\right)$ is non-sofic.

Proof. We merely sketch the proof of this theorem in order to show the parallels with the proof of Theorem 3.3 . The reader may fill in the details.

Let $M>0$ be given. Because $(\pi(Y), \mathbb{Z})$ has no synchronizing words, by Observation 4.3, the hypotheses of Theorem 2.3 are satisfied. Thus we can find $k=g K>0$, $m>M^{g}$ and occurring configurations $\left\{C_{i}\right\}_{i=1}^{m}$ on $B_{k}^{1} \subset \mathbb{Z}$ for which $\left\{E_{\pi(Y)}\left(C_{i}\right)\right\}_{i=1}^{m}$ is a union-increasing sequence.

Because $\left\{E_{\pi(Y)}\left(C_{i}\right)\right\}_{i=1}^{m}$ is a union-increasing sequence, we may choose points $\mathcal{S}=\left\{x^{(i)}\right\}_{i=1}^{m} \subseteq \pi(Y)$ with $x_{B_{k}^{1}}^{(i)}=C_{i}$ and

$$
x_{F_{k}^{1}}^{(i)} \notin E_{\pi(Y)}\left(C_{j}\right) \text { whenever } 1 \leq j<i \leq m .
$$

We will use this collection of points in $\pi(Y)$ to create a collection of points in $Y$ (and from these a collection of occurring configurations on $B_{k}^{2}$ ) as follows:

Let $\hat{y}^{(I)} \in \mathcal{S}^{K}$ be given by

$$
\hat{y}^{(I)}=\left(x^{\left(I_{1}\right)}, x^{\left(I_{2}\right)}, \ldots, x^{\left(I_{K}\right)}\right),
$$

where $\left(I_{1}, I_{2}, \ldots, I_{K}\right)$ is the $I^{t h}$ element in the lexicographic ordering on $\{1,2, \ldots$, $m\}^{K}$. Note that if $1 \leq J<I \leq m^{K}$ with $\ell$ the first index for which $J_{\ell} \neq I_{\ell}$, we have $J_{\ell}<I_{\ell}$ and

$$
x_{F_{k}^{1}}^{\left(I_{\ell}\right)} \notin E_{\pi(Y)}\left(x_{B_{k}^{1}}^{\left(J_{\ell}\right)}\right)=E_{\pi(Y)}\left(C_{J_{\ell}}\right) .
$$

Choose points $\left\{y^{(I)}\right\}_{I=1}^{m^{K}} \subseteq Y$ where the $(r g)^{t h}$ rows, $1 \leq r \leq K$, of $y^{(I)}$ are $\hat{y}^{(I)}$; that is, for all $1 \leq r \leq k$,

$$
\left.y^{(I)}\right|_{\mathbb{Z} \times\{r g\}}=\left.\hat{y}^{(I)}\right|_{\mathbb{Z} \times\{r\}} .
$$

Because $Y$ is block gluing in the $\vec{e}_{2}$-direction with gluing distance $g$, such a collection of points $\left\{y^{(I)}\right\}_{I=1}^{m^{K}}$ exists.

As in the proof of Theorem 3.3 , the collection of occurring configurations $\left\{y_{B_{k}^{2}}^{(I)}\right\}_{I=1}^{m^{k}}$ satisfies the hypotheses of Theorem 2.3 with $k=g K>0$ and $m^{k}>$ $\left(M^{g}\right)^{K}=M^{g K}=M^{k}$. Thus, $\left(Y, \mathbb{Z}^{2}\right)$ is non-sofic, as desired.

\section{REFERENCES}

[C] Cassaigne, J., Le Shift Impair Bidimensionnel est Sofique, Seminar Talk on September 30, 2010, http://www.lif.univ-mrs.fr/Sycomore/Julien-Cassaigne.html, accessed May 30, 2011.

[H] Hochman, M., private communication.

[HQ] Hoffman, C. and Quas, A., private communication.

[JKM] Johnson, A., Kass, S., and Madden, K., Projectional Entropy in Higher Dimensional Shifts of Finite Type, Complex Systems, 17 (2007), 243-257. MR2373706 (2008m:37028)

[LM] Lind, D. and Marcus, B., An Introduction to Symbolic Dynamics and Coding, Cambridge University Press, 1995. MR.1369092 (97a:58050)

[LMN] Lindgren, K., Moore, C., Nordahl, M., Complexity of Two-Dimensional Patterns, Journal of Statistical Physics, 91 (1998), 909-951. MR1637266 (2000d:68104)

[KM] Kass, S., and Madden, K., A Note on the Uniform Filling Property and Strong Irreducibility, Acta Applicandae Math. DOI 10.1007/s10440-013-9816-5 
[P] Pavlov, R., A Class of Nonsofic $\mathbb{Z}^{d}$ Shift Spaces, Proceedings of the American Mathematical Society, 141 (2013), 987-996. MR3003690

[P2] Pavlov, R., private communication.

[PS] Pavlov, R. and Schraudner, M., Projectional Subdynamics of $\mathbb{Z}^{d}$ Shifts of Finite Type (submitted).

[S] Schraudner, M., Projectional Entropy and the Electrical Wire Shift, Discrete and Continuous Dynamical Systems, 26 (2010), no. 1, 333-346. MR2552791(2010k:37027)

Department of Mathematics and Computer Science, Drew University, Madison, NEW JERSEY 07940

Department of Mathematics and Computer Science, Drew University, Madison, NeW Jersey 07940 